\title{
Updating Subjective Risks in the Presence of Conflicting Information: An Application to Climate Change
}

\author{
by \\ Trudy Ann Cameron* \\ Department of Economics, University of Oregon \\ and Department of Economics, UCLA
}

Keywords: risk elicitation, subjective probability, prior/posterior distributions, ambiguity aversion, Bayesian updating, alarmist learning

JEL Classifications: D8, N5, Q2

Address: Department of Economics, 435 PLC

University of Oregon, Eugene, OR 97403-1285

(541)346-1242 fax (541)346-1243

cameron@darkwing.uoregon.edu

Date: August 14, 2002 


\title{
Updating Subjective Risks in the Presence of Conflicting Information: An Application to Climate Change
}

\begin{abstract}
Willingness to support public programs for risk management often depends on individual subjective risk perceptions in the face of uncertain science. As part of a larger study concerning climate change, we explore individual updated subjective risks as a function of individual priors, the nature of external information, and individual attributes. We examine several rival hypotheses about how subjective risks change in the face of new information (Bayesian updating, alarmist learning, and ambiguity aversion). The source and nature of external information, as well as its collective ambiguity, can have varying effects across the population, in terms of both expectations and uncertainty.
\end{abstract}




\section{Updating Subjective Risks in the Presence of Conflicting Information: An Application to Climate Change}

When a new social problem emerges, people's preference orderings over possible policy solutions are initially uncertain and subject to frequent updating. For example, consider the evolving policy challenges posed by things like genetically modified organisms, human cloning, the Strategic Defense Initiative, biological weapons, species extinctions, the human carcinogenicity of new (or sometimes, old) substances, or the long-term storage of nuclear wastes. Information on the causes and/or potential consequences of these problems is often incomplete and contradictory. Over time, new and sometimes competing information emerges from various sources in ways that can change people's evaluations of the problem and therefore the appropriateness of different public policy responses.

The updating of subjective risks is also important to many private choices.

Government information programs are increasingly being implemented as partial remedies for market failure, since imperfect information is one of a number of excuses for such failures. ${ }^{1}$ But government information programs compete for the consumer's attention with other sources of information. ${ }^{2}$ The success of information programs, in terms of their effects on individual choices, thus also hinges on the processes whereby individuals update subjective risks. $^{3}$

In this paper, we use a particular example of a public policy problem--climate change risks. We focus on estimating the weights that individuals place on the opinions of government scientists and environmental groups as they update their subjective beliefs about the nature of this problem. We test four rival hypotheses about how people update their beliefs in the face of new and sometimes conflicting information. These hypotheses include (1) 
strict Bayesian updating, (2) alarmist learning, (3) ambiguity aversion, and (4) cross-sectional homogeneity/heterogeneity in the updating process.

For the climate change example, this research can be viewed as a precursor to understanding how willingness to pay to prevent climate change will evolve over time. ${ }^{4}$ How do people combine alternative information about future climate prospects, in terms of both expectation and uncertainty, based on evidence or conclusions that they pick up from different sources? Individuals will have different views about the credibility of these different sources, and sources deemed more-credible can be expected to have a greater influence.

In this paper, we focus on estimating the weights that individuals place on the opinions of government scientists and environmental groups in forming their opinions about future climate conditions in the absence of mitigation. The task is thus narrowly delineated. ${ }^{5}$ The quasi-experimental exercise we examine here is a stylized version of what goes on in the real world, but it is sufficient to explore richer models than have previously been examined for the process whereby individuals update their priors in response to external information. Our key results are demonstrated for a convenience sample, so further research is clearly warranted. Nevertheless, the present data produce some very clear findings concerning our four main hypotheses: people do not use strict Bayesian updating, they are not, on average, alarmist learners, but there is considerable ambiguity aversion and systematic heterogeneity in the manner in which different people respond to multiple alternative sources of climate information.

Section 1 provides a very simple model in the context of the climate change example and elaborates upon the four hypotheses. Section 2 describes the relevant portions of a survey instrument that was administered to a convenience sample of college students, resulting in a 
data set that allows some basic models, and their generalizations with heterogeneity, to be evaluated. Section 3 discusses the empirical results based on these data, which lead to two plausible models, each consisting of a system of nonlinear simultaneous equations with a variety of parameter restrictions, and Section 4 concludes with some speculation about how these new insights might be used.

\section{Updating Subjective Distributions based on Outside Information}

The cognitive process of updating subjective risks is a topic of intellectual merit on its own. However, we wish to emphasize in this paper that the relevant endpoint will typically concern how this updated risk information will alter public opinion about policies with respect to risk mitigation.

There has been substantial policy interest in recent years in the topic of risk communication (e.g. Davies et al., 1987). This literature focuses on the best way to convey to individuals the true objective magnitudes of risks. ${ }^{6}$ There has been less attention, however, to the problem of eliciting subjective probabilities. Reliable elicitation of (at least) the means and variances of the subjective probability distributions for climate chance consequences will be crucial to any analysis of society's willingness to incur the costs of climate change mitigation. $^{7}$

When risk perceptions feed into the demands for alternative public policies, nonmarket valuation via stated preference survey methods is often necessary. The "information effects" literature, within the broader literature on non-market valuation via survey methods, has concentrated on the extent to which information provided by the survey instrument affects the resultant estimates of willingness to pay (WTP) for non-market public goods (e.g. Blomquist and Whitehead (1998), Bohara et al. (1998), Munro and Hanley (1999), Hoehn and 
Randall (2002)). One of the key questions has concerned how much information should be provided to respondents in stated preference surveys. There must be enough detail to satisfy the recommendation of "full and unbiased information" (Arrow et al. 1993), but not so much as to create information overload and compromise survey response rates or respondent patience or comprehension (Bergstrom and Stoll, 1990, DeShazo and Fermo, 2002).

For most survey-based experiments in this vein, the strategy has been to vary the amount of information provided to respondents and then assess the effect of this information directly on WTP. It seems useful to decompose this overall effect into two constituent effects. New information first leads the individual to modify, to a greater or lesser extent, their prior impressions of the non-market public good for which they are being asked to pay. Subsequently, this change in perception affects their WTP for the good. In this paper, we attempt to provide greater resolution concerning the first of these two processes.

How do individuals update their prior subjective distributions for measures of future environmental quality as new information becomes available from sources that may have different levels of credibility in the mind of the individual? Hoehn and Randall (2002) devise a survey experiment that involves "treating" respondents with sequentially administered doses of information about each of a number of different dimensions of a natural resource injury. At each step, post-treatment injury perception is a weighted sum of prior information and the new information contained in an information treatment, but these weights are not heterogeneous across individuals. The emphasis in the Hoehn-Randall model is that one can allow heterogeneous baseline information to generate heterogeneous perceptions of resource injuries even though the informational treatment is the same. This is consistent with the observation in Cameron and Englin (1997) that survey-provided information is only incremental, added to 
whatever information survey subjects may have previously acquired on their own.

In this paper, the experimental design involves treating respondents with different information. We focus on the problem of climate change policy and consider estimates of future annual average temperatures, $t$ (simplifying greatly by treating climate change as a onedimensional problem, which it is not). Suppose individuals begin with a prior distribution for future climate in their region, $f(t)$, with expected value $E[t]_{o}$ and variance $V[t]_{o}$. We wish to model explicitly the data-generating process that yields each individual's revised values of $E^{*}[t]$ and $V^{*}[t]$ associated with their revised distribution $f^{*}(t)$ after they have been exposed to differing types of climate information attributed to different sources. These sources are characterized broadly as "government scientists" and "environmental groups."

Our survey instrument elicits subjective prior distributions for future average temperatures, provides all of the "outside" information (using a randomized design), and then elicits revised subjective distributions, so it is relatively simple to model the updating process. ${ }^{8}$ What hypotheses can be tested using these data? First, formal Bayesian updating strategies can be evaluated by testing strict Bayesian updating formulas for mean and precision against some more general specifications.

Second, it is appropriate to assess whether there is any evidence of "alarmist learning." Viscusi and Magat (1992) describe the possibility that individuals may overreact to information they receive. For what they term an alarmist learner, the relative informational weights on the two external sources of information are super-additive, meaning they would exceed one either individually or collectively. The discussion in this paper focuses on estimating the weights that individuals place on the opinions of government scientists and environmental groups. It will be possible to assess whether either of these weights is greater 
than one, or whether their sum exceeds unity.

Third, it is possible to adapt an idea of Viscusi and Magat (1992) to allow respondents' attentiveness to external information to be affected by the extent of the disparity among the sources of external information to which they are exposed. ${ }^{9}$ This disparity might be crudely summarized as the difference between the highest and lowest values (range, R) for each characteristic of the distribution of future average annual temperatures attributed to the different external information sources. If this range is small, the individual may place relatively greater trust in all outside sources than in his or her own prior subjective distributions. If the range is large, the respondent may retreat to place relatively greater weight on his or her own judgment. In the survey employed for this analysis, range $R$ in the outside opinions about $t$ is just another attribute of the array of external distributional characteristics that were part of the experimental design of the survey instrument. $R$ is therefore an exogenous variable. ${ }^{10}$

To determine whether the degree of ambiguity in the available external information has any systematic effect on the updating process, one would test the hypothesis that the coefficient(s) on the ambiguity term(s) is (are jointly) equal to zero. In a more elaborate model, where there might be a much larger number of outside information sources, the standard error of means across all outside sources might be a more useful measure of the degree of ambiguity. Whatever measure is used, it is associated only with the normalized weight on the individual's own prior subjective distribution on environmental quality. Whatever functional form is used to capture the ambiguity effect, it plays the same roleto shift the weight away from outside sources and towards the individual's prior subjective distribution. 


\section{Data: Survey and Sample}

The survey upon which this research is based was implemented, in part, as an extensive pre-test and pilot survey for a much larger study that addresses public support for a range of different climate change mitigation policies with different features. The later study, however, does not pose the same questions in the same way. ${ }^{11}$ The questions and answers analyzed here are unique to this pilot survey.

After two rounds of pretests, a pencil-and-paper questionnaire was distributed to Summer Session undergraduate economics classes. Students received a five-minute introduction to the survey during the lecture period and were requested to return the completed survey by the next lecture. ${ }^{12}$ A second round of sampling was conducted in two classes during the Fall quarter of the same year, and a third round of sampling, using similar classes, took place in the Fall of the next year. Table 1 gives descriptive statistics for the 608 subjects in the estimating sample.

For empirical assessment of risk perception in the context of climate change, information about subjective uncertainty over the entire trajectory of the full array of climate change consequences would be desirable. However, such trajectories are very difficult to elicit, so we begin with just a particular "snapshot": annual average temperature during a future decade.

Prior to the first elicitation of subjective future average temperature forecasts on the survey, actual historical annual data were provided for the weather station nearest the respondent. The data were depicted in the form of a scatter graph with annual average temperatures on the vertical axis (rounded to the nearest whole degree Fahrenheit) and years 
on the horizontal axis. A stylized thermometer, labeled "actual (1987-1996)" was positioned to the immediate right of this graph and the marginal distribution of average temperatures was shown as a horizontal histogram summarizing the variation in average annual temperatures over this decade. A second thermometer, labeled "average actual (1987-1996)" displayed an arrow showing the mean annual average temperature over this time interval (63.5 degrees). This incremental display strategy is intended to help the respondent understand that comparable summary information (annual averages, averaged over a decade) is being sought for a future decade. It also ensures that the respondent will be making his or her forecast for the expected value and dispersion of future mean temperatures based on valid current data.

On the facing page of the survey instrument, we then elicited information on the mean and dispersion of the individual's prior subjective distribution for future environmental quality. As an anchoring device, the thermometer showing the historical mean annual average temperature was re-displayed each time any temperature information was elicited or provided.

To elicit a subjective distribution for future annual average temperatures, the survey used a pair of thermometer scales, labeled "best guess future (2011-2020)" and "high, low guesses future (2011-2020)". Respondents were instructed to "use arrows by each thermometer" to convey information about expected value and uncertainty about annual average temperatures in their region in the decade of 2011-2020. Expected values seem relatively easy to elicit. It is more difficult to ask respondents to convey information on variances. For dispersion measures, we elected to ask individuals for their "high guess and low guess (meaning that there is only a $5 \%$ chance that the average temperature will be outside this interval)." We then interpret this range as roughly four standard deviations, squaring 0.25 times this amount to yield a variance approximation. Of course, this strategy ignores any 
asymmetry in this distribution.

Once these prior distributions have been established, the respondent is presented with information describing the distributions of future average temperatures (purportedly) forecasted by government scientists and by environmental groups. This was done overleaf in the survey instrument, and after the respondent had broken a seal that inhibited access to the next (centerfold) page of the survey booklet. ${ }^{13}$ The reference data thermometer was presented again, but this time there were two additional pairs of thermometers. For each of "Government Scientists" and "Environmental Groups", we depict separate thermometers for "most likely future (2011-2020)" and "high; low future (2011-2020)" guesses. ${ }^{14}$

Respondents appeared to have no difficulty understanding what was required of them. A key objective of the analysis, of course, is to discriminate among the effects of different external information sources on the respondent's distributional updating process. The design of the different survey versions ensures that there is orthogonal variation across respondents in these purported external forecasts. (See the Appendix.)

On a facing page, after viewing the external information on future climate, respondents are invited to use a final pair of thermometers to update their priors on the distribution of future annual average temperatures, giving both a new expected value and a new $95 \%$ range (which we again convert to a variance, invoking the same strong distributional assumptions).

Each temperature scale in the survey was continuous, and one of the tasks during data entry was to impute as closely as possible, to one decimal place on the temperature scale, the position of the point of each arrow drawn by each respondent. The analysis in this paper involves the information on the four pairs of temperature scales: the subjective prior distribution entered by the respondent, the stylized external information about future 
temperature distributions depicted by that particular survey variant for "Government Scientists" and for "Environmental Groups" (there were sixteen unique combinations), and the updated subjective posterior distribution entered by the respondent. It is this last updated distribution that this research seeks to explain, using the other three distributions and an array of sociodemographic and attitudinal variables. Summary statistics for the estimating sample are presented in Table 1.

\section{Empirical Findings}

How do survey respondents process the information attributed to government scientists and environmental groups when they are given the opportunity to update their own opinions in the light of this additional information? One possibility is that they may be Bayesians. If so, their own subjective prior distribution and different pieces of external information are all ingredients in the formulation of an updated distribution, but a very precise set of weights is involved. These weights reflect the uncertainty surrounding each constituent piece of information. In the presence of two additional sources of information about mean and variance (precision) of a distribution, the usual textbook Bayesian formulas for the case of one additional source appear to generalize straightforwardly: the posterior mean should be a precision-weighted average of the component means, and the posterior precision should be the sum of the component precisions. ${ }^{15}$

In order to simplify the notation, define the sum of precisions:

$$
D=\left(V[t]_{o}\right)^{-1}+\left(V[t]_{g}\right)^{-1}+\left(V[t]_{e}\right)^{-1}
$$

where the subscript "o" on each variance term stands for "own," the subscript "g" stands for "government scientists," and the subscript "e" stands for "environmental groups." Similar 
subscripting will modify expectations and other descriptors of the different distributions of future temperatures. It seems appropriate to test whether the updating process could be Bayesian, against the alternative that it is more general, by specifying the following two regression models, one for the posterior expected value, and one for the posterior precision:

$$
\begin{gathered}
E^{*}[t]=\alpha_{0}+\alpha_{1} \frac{\left(V[t]_{o}\right)^{-1} E[t]_{o}}{D}+\alpha_{2} \frac{\left(V[t]_{g}\right)^{-1} E[t]_{g}}{D}+\alpha_{3} \frac{\left(V[t]_{e}\right)^{-1} E[t]_{e}}{D}+\varepsilon_{E} \\
V^{*}[t]^{-1}=\beta_{0}+\beta_{1}\left(V[t]_{o}\right)^{-1}+\beta_{2}\left(V[t]_{g}\right)^{-1}+\beta_{3}\left(V[t]_{e}\right)^{-1}+\varepsilon_{P}
\end{gathered}
$$

Estimating these two models, and testing the joint hypothesis that $\alpha_{0}=0$ and $\alpha_{1}=\alpha_{2}=\alpha_{3}=1$ for the $E^{*}[t]$ model and the joint hypothesis that $\beta_{0}=0$ and $\beta_{1}=\beta_{2}=\beta_{3}=1$ for the $V^{*}[t]^{-1}$ model (either separately, or jointly) would constitute a rough test of whether the updating process could indeed be strictly Bayesian.

The first and third columns in Table 2 shows the results for unrestricted estimation of the generalized Bayesian models in equations (2) and (3), and the second and fourth columns show analogous estimates when the intercepts are constrained to zero. While the unrestricted point estimates of the slope parameters appear close to unity in the case of $E^{*}[t]$ (for equation (2) ), F-tests of the restriction that all slopes are simultaneously one soundly reject that hypothesis. (The P-values for these F-tests are essentially zero for the $E^{*}[t]$ equation.) For $V^{*}[t]^{-1}$ (equation (3)), the point estimates of the individual slopes are markedly different from one, and the null hypothesis of jointly unitary slopes is again rejected conclusively. These individuals are not behaving like strict Bayesians.

A competing model might be that respondents' updated expected values $E^{*}[t]$ and 
updated variances $V^{*}[t]$ are determined more simply. Instead of the more elaborate general linear function of the precision-weighted expectations in equation (2), used for testing Bayesian updating, consider the hypothesis that $E^{*}[t]$ is simply a linear function of $E[t]_{o}$, $E[t]_{g}$, and $E[t]_{e}$ :

$$
E^{*}[t]=\alpha_{0}^{\prime}+\alpha_{1}^{\prime} E[t]_{o}+\alpha_{2}^{\prime} E[t]_{g}+\alpha_{3}^{\prime} E[t]_{e}+\varepsilon_{E}^{\prime}
$$

One can test the two competing non-nested hypotheses in equations (2) and (4) with a Cox test and a Davidson-MacKinnon J-test. These tests can be applied for specifications with and without intercepts. For specifications of $E^{*}[t]$ with and without intercept terms, the Cox tests and the J-tests reject both the model in equation (2) and that in equation (4).

For dispersion, analogous Cox and J-tests are not appropriate. Again, the competing ad hoc model should probably consist of a simple linear relationship between $V^{*}[t]$ and the constituent variances, $V[t]_{o}, V[t]_{g}$, and $V[t]_{e}$ :

$$
V^{*}[t]=\beta_{0}^{\prime}+\beta_{1}^{\prime} V[t]_{o}+\beta_{2}^{\prime} V[t]_{g}+\beta_{3}^{\prime} V[t]_{e}+\varepsilon_{V}^{\prime}
$$

The dependent variable in the generalized Bayesian specification in equation ( 3 ), however, is the posterior precision, not the posterior variance (unless nonlinear-in-parameters models are broached) so the competing variance specifications are not as amenable to Cox or J-tests.

This preliminary evidence seems to suggest quite clearly, however, that the strict Bayesian formulation is probably not appropriate in this context, particularly for the variance. The simple linear model appears to dominate even the generalization of the Bayesian specification with nonzero intercept and non-unitary slopes. So it is reasonable to consider possible ad hoc specifications more carefully.

In Table 3, the first two models we tabulate are for $E^{*}[t]$, the respondent's revised 
subjective average annual temperature. The first column displays results for the unrestricted linear function in equation (4) and the second column shows the slope estimates when the intercept in this specification is restricted to zero. (This intercept restriction can be rejected at the $5 \%$ level according to the t-test statistic on the intercept in the first model, which does not allow for respondent heterogeneity. In models with heterogeneity, like the ones we will ultimately prefer, this restriction is not rejected). If we impose the intercept restriction in this simple model with constant parameters, we cannot reject the further restriction that the sum of the slopes is unity. The individual slope coefficients in both models are all strongly statistically significant.

The model with the intercept restriction is offered in order to test for the presence of Viscusi-Magat "alarmist learning." With a zero intercept imposed, but the slope parameters free to take on whatever values are dictated by the data, the P-value for the F-test statistic for the null hypothesis that the sum of the slopes on $E[t]_{g}$ and $E[t]_{e}$ is equal to one is 0.377 , which fails to reject the hypothesis. The confidence interval for the sum of the slopes on $E[t]_{g}$ and $E[t]_{e}$ includes values both less than and greater than one. There is no suggestion, therefore, that the coefficients on the outside information are greater than one either individually, or in sum, so there is no statistical evidence of alarmist learning with respect to $E^{*}[t]$ in this sample.

Other preliminary models (not tabulated) explore the hypothesis that the extent of the disparity between the two outside sources of information about the $E[t]$ will affect the weight applied to $E[t]_{o}$, the own-prior. In an otherwise unrestricted specification, the greater the ambiguity in the outside information (the greater the disparity between $E[t]_{g}$ and $E[t]_{e}$ ), the 
greater is the weight on the own-expectation, and by a statistically very significant amount. Also in these models, the intercept coefficient becomes insignificantly different from zero at the $5 \%$ level. For subsequent modeling efforts concerning $E^{*}[t]$, based on this accumulated evidence, we will be using a model with zero intercept, ambiguity effects, and weights on own prior and outside information that sum to unity—generalized to include systematically varying parameters. This choice is also driven partly by its expedience for the potential application of simulating a counterfactual shift to zero weights on specified information sources, while preserving the relative weights on the other sources.

Table 3 also explores some simple linear specifications for the relationship between posterior variance $V^{*}[t]$ and the three component variances, as in equation (5). The unrestricted linear specification in the first model shows the intercept to be insignificantly different from zero. The second model restricts the intercept to zero and forces the slopes to sum to unity. This set of two restrictions cannot be rejected. Additional models (not tabulated) reveal that ambiguity effects are also important in ad hoc models to explain posterior variance. When the slope on the own prior variance is allowed to shift with the magnitude of the disparity between the two outside sources, the positive slope differential bears a t-test statistic in excess of eleven.

Based on these preliminary models, we will be assuming that both expected values and variances can be reasonably well approximated by simple weighted averages of the three components, provided ambiguity effects are recognized. These models will be the basis for the generalizations to be described next.

\subsection{Two-equation model with homogeneous ambiguity effects}


In Table 4, we show the results of employing the available variables capturing respondent characteristics as factors that may shift an individual's relative weights on the different sources of information about future climate conditions (for both the mean and the variance). The estimates stem from a two-equation simultaneous non-linear least squares (NLLS) model that is specified in a manner that ensures that the models display zero intercepts and non-negative weights that sum to unity, for both the expectation equation and the variance equation. The basic form of the jointly estimated model is:

$$
\begin{aligned}
E^{*}[t]=\left(1+\kappa_{E} \mid E[t]_{g}\right. & \left.-E[t]_{e}\right)\left[\left(1+\kappa_{E}\left|E[t]_{g}-E[t]_{e}\right|\right)+\exp \left(\gamma_{g}\right)+\exp \left(\gamma_{e}\right)\right]^{-1} E[t]_{o} \\
& +\exp \left(\gamma_{g}\right)\left[\left(1+\kappa_{E}\left|E[t]_{g}-E[t]_{e}\right|\right)+\exp \left(\gamma_{g}\right)+\exp \left(\gamma_{e}\right)\right]^{-1} E[t]_{g} \\
& +\exp \left(\gamma_{e}\right)\left[\left(1+\kappa_{E}\left|E[t]_{g}-E[t]_{e}\right|\right)+\exp \left(\gamma_{g}\right)+\exp \left(\gamma_{e}\right)\right]^{-1} E[t]_{e}+\varepsilon_{E} \\
V^{*}[t]=\left(1+\kappa_{V} \mid V[t]_{g}\right. & \left.-V[t]_{e} \mid\right)\left[\left(1+\kappa_{V}\left|V[t]_{g}-V[t]_{e}\right|\right)+\exp \left(\delta_{g}\right)+\exp \left(\delta_{e}\right)\right]^{-1} V[t]_{o} \\
& +\exp \left(\delta_{g}\right)\left[\left(1+\kappa_{V}\left|V[t]_{g}-V[t]_{e}\right|\right)+\exp \left(\delta_{g}\right)+\exp \left(\delta_{e}\right)\right]^{-1} V[t]_{g} \\
& +\exp \left(\delta_{e}\right)\left[\left(1+\kappa_{V}\left|V[t]_{g}-V[t]_{e}\right|\right)+\exp \left(\delta_{g}\right)+\exp \left(\delta_{e}\right)\right]^{-1} V[t]_{e}+\varepsilon_{V}
\end{aligned}
$$

where $\left(\varepsilon_{E}, \varepsilon_{V}\right) \sim B V N(0, \Sigma)$.

The six different parameters, $\kappa_{E}, \kappa_{V}, \gamma_{g}, \gamma_{e}, \delta_{g}$ and $\delta_{e}$ can each be estimated as a single scalar, analogous to the independently estimated equations in Table 3 with all restrictions imposed. Instead, we have reparameterized each equation to incorporate the restrictions and then rendered each of the $\gamma \mathrm{s}$ and $\delta \mathrm{s}$ a systematic varying parameter. We employ as potential shifters for all of these four parameters (i.) some available sociodemographic variables (age, gender), as well as (ii.) subjective characteristics (informedness about environmental issues, degree of conservatism, likelihood of living in the same area in the future, and perceptions of researcher bias), and (iii.) dummy variables to 
identify observations collected in the second and third subsamples. Not all of these variables are statistically significant shifters of all four of the systematic varying parameters in this specification. Persistently insignificant terms have therefore been dropped.

The model in Table 4 assumes cross-sectionally constant Viscusi-Magat ambiguity effects. Each of the $E^{*}[t]$ and $V^{*}[t]$ equations in the joint model is augmented with a single ambiguity parameter, $\kappa_{\mathrm{E}}$ and $\kappa_{\mathrm{V}}$, respectively, that allows the weight placed on the individual's own priors to vary systematically with the absolute difference between the two external opinions. These estimated ambiguity effect parameters are displayed as the first column in each triple of columns in Table 4. Both of these coefficients are positive, as anticipated, and strongly statistically significant. The greater the disparities in the outside opinions about either of the moments of the future temperature distribution, the greater the weight placed on the respondent's own priors. ${ }^{16}$

The other implications of the estimated model in Table 4 also seem plausible. First, while it is not possible to distinguish an age effect from a cohort effect in an essentially crosssectional sample such as this, it nevertheless appears that older college students place less weight on environmental group opinions in deciding upon $E^{*}[t]$, and less weight on government scientists in deciding upon $V^{*}[t]$.

Gender is the only variable to have a statistically significant effect on all four weighting parameters. In formulating both $E^{*}[t]$ and $V^{*}[t]$, females in our sample place relatively more weight on the opinions of government scientists and environmental groups than do males. In short, males appear to "know what they know." This stands in contrast to the results of Siegrist and Cvetkovich (2001), who find that female students had less trust in authorities concerning the random nature of cancer clusters and perceived a cancer cluster "as 
less likely to be a result of pure chance than did males." There is, however, evidence of asymmetry about trust in authorities concerning good and bad news. Perhaps females are more trusting of bad news in the form of climate change risks than they are of good news in the form of cancer clusters being purely random. ${ }^{17}$

Gender differences in risk perception have been identified by Finucane et al. (2000), who elaborate upon earlier findings by Flynn et al. (1994) that race and gender differences in risk perception in the US were primarily due to that $30 \%$ of the white male population who judge risks to be extremely low. We have not focused in this paper on the determinants of initial risk perception (namely, $E[t]_{o}$ and $V[t]_{0}$ ). However, it is indeed the case that when the own-prior expectations, $E[t]_{o}$, are regressed linearly on the same characteristics that we used to explain attention to each outside authority, gender is the only variable with an individually statistically significant effect. Females' prior expected future average temperatures are higher by 0.44 degrees Fahrenheit than are those for males (at the $10 \%$ level). $V[t]_{0}$ is also systematically higher for females than for males, by a statistically significant 0.6462 degreessquared. (Conservatism, which decreases prior subjective future temperature variances, is the only other marginally significant factor.)

Returning to the results in Table 4, a greater subjective degree of informedness about environmental issues decreases the relative weight on government scientists in formulating $E^{*}[t]$. It does not appear to have a significant effect upon any of the other weights.

Students who identify themselves as being more conservative appear to place systematically more weight on government scientists' opinions about $E^{*}[t]$ than do moreliberal students, but less weight on government scientists' opinions about $V^{*}[t]$. The more 
likely the student is to remain in the same region in the future, the more weight they tend to place on government scientists opinions in formulating their assessments of $E^{*}[t]$ (but not $V^{*}[t]$ ). (As an alternative interpretation, the more likely it is that they will move to another region before the future time period stipulated in the survey, the less attention they pay to government opinions about $E^{*}[t]$ for their current region.)

Concerning researcher bias, the more the research team is perceived to be biased in favor of climate change mitigation programs, the less is the weight assigned to the ostensible government information sources in formulating an opinion about $E^{*}[t]$. However, perceptions of researcher bias have no statistically discernible effects on the weights assigned to different information sources in formulating a revised subjective variance, $V^{*}[t]$.

The dummy variables for the second and third subsamples will be picking up all systematic differences between enrollees in the courses from which the observations are drawn and also any other differences in their full information sets. The coefficients on these dummy variables could be capturing much more than just a simple year-to-year cohort effect. With these caveats, and compared to the first subsample, respondents in the second subsample appear to be less attentive to environmental groups' opinions about $E^{*}[t]$. In contrast, the third subsample appears to be less attentive to government scientists' opinions. Again, these differences may reflect many things, including different weather patterns during or prior to each sampling window.

\subsection{Three-equation Model with Heterogeneous Ambiguity Effects}

The 95\% range information directly elicited from respondents was converted into a 
proxy for the anticipated variance in uncertain future temperatures (by imposing a strong assumption about symmetry) in order to test hypotheses about Bayesian-type behavior. Since the Bayesian model appears to be dominated by more-general specifications, we now consider specifications wherein different updating processes are permitted for not only $E^{*}[t]$, but separately for the "high guess" and "low guess" updated predictions as well. We will call these updated predictions $H^{*}[t]$ and $L^{*}[t]$. This leads to a three-equation analog to the system of equations in (6) and (7). When the two new equations are used in lieu of the single variance equation, there are different sets of parameters in play. $\kappa_{H}$ and $\kappa_{L}$ are now the Viscusi-Magat ambiguity parameters for the high-guess and low-guess equations. For the high-guess equation, $\lambda_{g}$ and $\lambda_{e}$ will be the weighting parameters for the external information from government scientists and environmental groups, and $\theta_{g}$ and $\theta_{e}$ will be the corresponding weighting parameters for external low-guess values. The revised system of equation is:

$$
\begin{gathered}
\begin{aligned}
E^{*}[t]=\left(1+\kappa_{E} \mid E[t]_{g}\right. & \left.-E[t]_{e}\right)\left[\left(1+\kappa_{E}\left|E[t]_{g}-E[t]_{e}\right|\right)+\exp \left(\gamma_{g}\right)+\exp \left(\gamma_{e}\right)\right]^{-1} E[t]_{o} \\
+ & \exp \left(\gamma_{g}\right)\left[\left(1+\kappa_{E}\left|E[t]_{g}-E[t]_{e}\right|\right)+\exp \left(\gamma_{g}\right)+\exp \left(\gamma_{e}\right)\right]^{-1} E[t]_{g} \\
+ & \exp \left(\gamma_{e}\right)\left[\left(1+\kappa_{E}\left|E[t]_{g}-E[t]_{e}\right|\right)+\exp \left(\gamma_{g}\right)+\exp \left(\gamma_{e}\right)\right]^{-1} E[t]_{e}+\varepsilon_{E} \\
H^{*}[t]=\left(1+\kappa_{H} \mid H[t]_{g}\right. & \left.-H[t]_{e} \mid\right)\left[\left(1+\kappa_{H}\left|H[t]_{g}-H[t]_{e}\right|\right)+\exp \left(\lambda_{g}\right)+\exp \left(\lambda_{e}\right)\right]^{-1} H[t]_{o} \\
& +\exp \left(\lambda_{g}\right)\left[\left(1+\kappa_{H}\left|H[t]_{g}-H[t]_{e}\right|\right)+\exp \left(\lambda_{g}\right)+\exp \left(\lambda_{e}\right)\right]^{-1} H[t]_{g} \\
& +\exp \left(\lambda_{e}\right)\left[\left(1+\kappa_{H}\left|H[t]_{g}-H[t]_{e}\right|\right)+\exp \left(\lambda_{g}\right)+\exp \left(\lambda_{e}\right)\right]^{-1} H[t]_{e}+\varepsilon_{H} \\
L^{*}[t]=\left(1+\kappa_{L} \mid L[t]_{g}\right. & \left.-L[t]_{e}\right)\left[\left(1+\kappa_{L}\left|L[t]_{g}-L[t]_{e}\right|\right)+\exp \left(\theta_{g}\right)+\exp \left(\theta_{e}\right)\right]^{-1} L[t]_{o} \\
+ & \exp \left(\theta_{g}\right)\left[\left(1+\kappa_{L}\left|L[t]_{g}-L[t]_{e}\right|\right)+\exp \left(\theta_{g}\right)+\exp \left(\theta_{e}\right)\right]^{-1} L[t]_{g} \\
+ & \exp \left(\theta_{e}\right)\left[\left(1+\kappa_{L}\left|L[t]_{g}-L[t]_{e}\right|\right)+\exp \left(\theta_{g}\right)+\exp \left(\theta_{e}\right)\right]^{-1} L[t]_{e}+\varepsilon_{L}
\end{aligned}
\end{gathered}
$$


where $\left(\varepsilon_{E}, \varepsilon_{H}, \varepsilon_{L}\right) \sim M V N(0, \Sigma)$.

The NLLS estimates for systematically varying versions of the parameters in this system are presented in Table 5. Note that in addition to replacing the variance equation by separate equations for the "high guess" and "low guess" about future temperatures, we generalize the model by allowing all three ambiguity parameters, $\kappa_{E}, \kappa_{H}$ and $\kappa_{L}$, not just the weighting parameters, to vary with measured respondent attributes. This appears to be the first empirical attempt to identify heterogeneity in the degree of ambiguity aversion across subjects. For $E^{*}[t]$, for the base category of environmentally uninformed males, ambiguity in outside information increases reliance on own prior opinions. This effect is significantly larger for females, but for more informed individuals, the ambiguity effects shrink.

For the $E^{*}[t]$ equation, as we move from the two-equation model to the three-equation model, there are some adjustments to the set of variables which have statistically significant effects on respondents' "best guess" about future temperatures. However, coefficients that are significant in both specifications retain the same signs.

The weight placed on the opinions of government scientists about expected future mean temperatures is no longer significantly affected by the respondent's degree of conservatism in the three-equation model, and the effect of his or her perception of researcher bias in favor of climate change mitigation programs becomes insignificant as well. But other variables that did not make individually statistically significant contributions to the weights in the two-equation model now figure significantly in the three-equation model. Greater age (in my 17- to 23-year-old sample) now decreases reliance on government scientists' opinions in forming expectations about future climate. The second subsample (SMPL2) is also 
statistically significantly different in terms of the weights it appears to place, on average, on the opinions of government scientists. In the original two-equation model, degree of subjective informedness about environmental issues was not a significant determinant of the weight placed on the opinions of environmental groups. In the three-equation model, greater subjective informedness significantly reduces the attention paid to environmental groups. ${ }^{18}$

For $H^{*}[t]$, the intercept in the ambiguity aversion term is statistically significantly negative. However, for the age levels represented in this sample, the fitted value of the ambiguity aversion term will be positive. It will also decrease with the respondent's degree of conservatism.

Not surprisingly, the variables that affected the weights on government scientists and environmental groups opinions about "variance" also affect the weights on these same opinions about the "high guess." Some additional weight coefficients become significant, however, when the information about high and low guesses is not combined into an estimate of variance. Greater informedness now reduces reliance on government scientists' opinions in forming a revised opinion about the likely upper bound on average temperatures. The greater his or her perceived bias of the research team in favor of climate change mitigation programs, the less weight the respondent places on the ostensible opinions of government scientists. Greater conservatism reduces reliance not only on government scientists' opinions about the upper bound, but also on environmental groups opinions about this upper bound. The second subsample places significantly less weight on both government scientists and environmental groups opinions than the rest of the sample.

There is rather less statistically significant heterogeneity in the weighting process that yields respondents' revised opinions about the lower bound of the range for future average 
temperatures in this region. However, the design of the outside opinions about the lower bound exhibits much less variability than the design for the means and the upper bounds (see Table 1), so perhaps this lesser measured sensitivity is not surprising.

For the lower bound equation, greater disparity between the outside sources actually seems to decrease reliance on one's own priors in formulating opinions, at least for females. Older respondents place less weight on government scientists' opinions, but females place more weight on these opinions. Neither age nor gender has a statistically significant effect on the weight placed on environmental groups' opinions, but greater informedness about environmental issues or greater conservatism both reduce reliance on the opinions of environmental groups about the lower bound, which is unsurprising. Both the second and third subsamples display a lower average weight on the opinions of environmental groups about the lower bound on future local average temperatures.

\subsection{Other potential specifications}

Hoehn and Randall (2002) reiterate the findings of Viscusi and O'Connor (1984) and Viscusi (1989) showing, in a Bayesian model, that new information has an effect if it differs from subjects' prior knowledge. We have explored a more-general specification wherein the vector of individual attributes used to shift the weights placed on the opinions of government scientists and environmental groups in each of equations (8)- (10) is augmented by a measure of the difference between the respondents own prior and the corresponding opinion of each source. For example, the systematically varying weighting parameter on government scientists' opinions about future annual average temperatures gains a term in $E[t]_{g}-E[t]_{o}$ and the weight on environmental groups gains a term in $E[t]_{e}-E[t]_{o}$. Unfortunately, if we retain 
the terms for ambiguity effects in the model, which involve the differences between the two outside information sources' opinions, the nonlinear optimization process cannot be induced to converge. This is undoubtedly due to there being too much collinearity between the external information ambiguity term, $E[t]_{g}-E[t]_{e}$, and the two difference-from-own-prior terms, $E[t]_{g}-E[t]_{o}$ and $E[t]_{e}-E[t]_{o}$.

\section{Conclusions}

The limited size of the convenience sample used for the empirical portion of this study $(\mathrm{n}=608)$, means that the findings from the analysis of this survey are not conclusive. They are, however, provocative. The issue of how individuals process the disparate information they receive concerning risks for which the underlying science is uncertain has not been addressed in any such detail before. The process is likely to be similar across different risks, even though we have in this paper worked with an empirical example concerning climate change risks.

What has been learned about the evolution of public opinion in the face of new information by examining the nature of one such process at an individual level?

1. People do not appear to be strict Bayesians, although they do seem to achieve their updated opinions by using weighted averages of their own prior opinions and whatever outside information becomes available. The nature of these weights, however, varies across individuals.

2. There is no evidence of alarmist learning, at least on average, in this sample of 608 survey participant drawn from a college population. This does not preclude occasional instances of alarmist learning on the part of particular individuals, but any such 
behavior is too rare to appear in these data.

3. There can be substantial and significant ambiguity effects. Disagreement among external information sources decreases the amount of attention paid to them by individuals as they update their priors and the extent of this effect varies significantly across the population. For some stakeholder groups, this insight can help target campaigns of obfuscation to the constituencies that are most vulnerable.

For researchers designing the "information matter" to present to respondents in stated preferences studies, the lesson is that it can matter greatly whether this information is characterized as controversial or consensus opinion, and to different extents for different subpopulations. The usual goal of such studies is to value a common objective risk for all respondents. Prior subjective risks are rarely elicited, and the risk assumptions that motivate people's policy choices are presumed to coincide exactly with the information matter provided in the survey instrument. This coincidence is unlikely ever to be perfectly achieved, but it seems clear that the match will be closest if the information matter can be portrayed as a broad scientific consensus.

4. The source of information can have an effect on subjective risk that will vary across types of respondents.

a. First, my limited sample of 17- to 23-year-old college students does not allow us to get a complete picture of the age profile of susceptibility to outside information sources in formulating opinions about climate change. However, the fact that there are statistically significant disparities in the opinion-updating process by age, even within this very narrow age range, suggests that there may 
be important age effects in the general population as well. In any strategy to manipulate public support for mitigation programs via the provision of information, the sources and targeting of information by age group is likely to be important.

b. Second, my results suggest that women (at least young women) may be far more susceptible to the prognostications of perceived experts than are (young) men. Gender-targeted information campaigns may have a more cost-effective impact on public support for climate change mitigation policies than generic campaigns. Furthermore, gender appears to have a similar effect on the amount of attention paid to both types of outside information sources in this study.

However, the greater the disparity between the competing outside information sources (i.e. the informational ambiguity), the greater the weight that everyone in my sample tends to place on their own prior opinions, at least with respect to mean predictions about climate change. Per degree Fahrenheit of disparity between the two outside information sources, the tendency to adhere to one's own prior opinions about mean climate change is $80 \%$ greater for women than for men. Thus, to have the maximum effect on the audience, (and especially on women) any information campaign should be consistent across authorities.

c. Third, campaigns to increase individuals' perceptions of themselves as being well-informed about environmental issues will induce them to pay less attention both to government scientists and to environmental groups, and to rely instead on their own opinions about climate change prospects. Provided these own 
opinions are valid (i.e. provided individuals actually are better informed about environmental issues), this decrease in attention paid to the opinions of outside "expert" sources when new information is provided may be effectively offset.

d. An increased degree of conservatism, as may accompany different political regimes, may decrease the attention paid to both government scientists' and environmental groups' opinions about the upper bounds on possible climate change. Thus, information campaigns to raise awareness about climate change prospects will be less successful in a more conservative political climate.

However, greater conservatism will also tend to reduce the extent to which disparities in these outside information sources lead the individual to revert to their own priors in formulating a revised subjective estimate of the upper bound on mean temperature changes. This tendency may lead environmental groups, for example, to become more strident in their calls for climate mitigation policy during conservative regimes, partly by exaggerating the likely climate prospects and consequences in the absence of mitigation. Less attention would be paid to them otherwise, and the loss of attention from being out of line with government scientists' predictions is diminished compared to what it would be in a more liberal political environment.

The models explored in this paper were developed because it is clear from other work (Cameron, 2002) that individuals' subjective perceptions of climate change risks at the point of making a policy choice figure strongly in their willingness to pay for climate change mitigation programs. Rather than taking these risk perceptions as exogenously given, we allow in this analysis for the fact that they can be manipulated by available information and we 
uncover a surprising degree of statistically significant heterogeneity in the way individuals appear to process risk information under conditions of uncertainty. With a larger and more representative sample from the general population, richer models could almost certainly be supported. The robustness of these findings across other types of risks should also be evaluated.

For the climate change problem, willingness to pay for climate change mitigation programs constitutes willingness to pay for a substantial public good, and there are few indirect markets that can allow us to infer the needed demand information from revealed preferences. Consequently, stated preference techniques are likely to prove essential to any attempts at comprehensive valuation of climate change mitigation benefits and the benefits from reducing other poorly understood risks. Any stated preference exercise must be shown to pass a "scope test," wherein willingness to pay for the good varies directly with the amount of the good at stake (Arrow et al. 1993). The amount of the good at stake- "risk reduced"-in many of these cases is subjective and highly uncertain for most consumers. Thus, an understanding of subjective distributions for these risks - both in terms of expectation and the degree of uncertainty — and how these subjective distributions can be influenced, either naturally or strategically, will be critical to the public decision-making process for many challenging public policy problems. This study contributes to such an understanding. 
Table 1

Descriptive Statistics for Complete Estimating Sample $(\mathrm{n}=608)$

\begin{tabular}{llll}
\hline VARIABLE & DESCRIPTION & MEAN & STD. DEV \\
\hline
\end{tabular}

Distributions of future annual mean temperature: (degrees Fahrenheit)

$\begin{array}{clll}E[t]_{o w n} & \text { Prior subjective mean temp. } & 66.58 & 2.81 \\ E[t]_{g o v} & \text { "Government Scientists" mean temp. } & 66.03 & 0.50 \\ E[t]_{e n v} & \text { "Environmental Groups" mean temp. } & 68.15 & 1.49 \\ E^{*}[t] & \text { Posterior subjective mean temp. } & 66.74 & 1.88 \\ V[t]_{o w n} & \text { Prior subjective temp. "variance" } & 2.71 & 3.78 \\ V[t]_{g o v} & \text { "Government Scientists" temp. "variance" } & 1.12 & 0.82 \\ V[t]_{e n v} & \text { "Environmental Groups" temp. "variance" } & 4.12 & 3.44 \\ V^{*}[t] & \text { Posterior subjective temp. "variance" } & 2.23 & 2.30 \\ H[t]_{o w n} & \text { Prior subjective "high guess" for temp. } & & \\ H[t]_{g o v} & \text { "Government Scientists" "high guess" for temp. } & 69.43 & 3.81 \\ H[t]_{e n v} & \text { "Environmental Groups" "high guess" for temp. } & 67.90 & 1.09 \\ H{ }^{*}[t] & \text { Posterior subjective "high guess" for temp. } & 71.37 & 2.92 \\ L[t]_{o w n} & \text { Prior subjective "low guess" for temp. } & 69.60 & 2.61 \\ L[t]_{g o v} & \text { "Government Scientists" "low guess" for temp. } & 63.83 & 2.70 \\ L[t]_{e n v} & \text { "Environmental Groups" "low guess" for temp. } & 64.16 & 0.65 \\ L^{*}[t] & \text { Posterior subjective "low guess" for temp. } & 64.56 & 1.22 \\ & & 64.18 & 1.73\end{array}$

Respondent Attributes:

AGE Age of respondent (17- to 23-year-old sample)

FEMALE

INFORM

CONSRV

IMMOBILE

RES. BIAS

SMPL2

SMPL3
Gender $(0=$ male, $1=$ female $)$

"I consider myself well-informed about environmental Issues" ( 1 =strongly disagree, 7 =strongly agree $)$

"I consider myself to be..."

( 1 = extremely liberal, 7 = extremely conservative $)$

"Fifteen to twenty years from now, I expect to live in the Same region as I do now."

$(1=$ strongly disagree, $7=$ strongly agree $)$

“...I suspect that the [team] conducting this study believes that preventing climate change is...

( 1 = extremely unnecessary, 7 =extremely urgent )

Membership in Fall 1997 subsample

Membership in Fall 1998 subsample
19.39

0.5065

4.072

3.952

1.22

4.295

1.72

5.267

1.13

0.3898

0.4967 
Table 2: Test of Bayesian Updating in Formulation of Posterior Subjective Distribution of Future Annual Average Temperatures $(n=608)$

\begin{tabular}{|c|c|c|c|c|}
\hline \multirow{2}{*}{$\begin{array}{l}\text { VARIABLE }{ }^{\mathrm{a}} \\
\text { constant }\end{array}$} & \multicolumn{2}{|c|}{$E^{*}[t]:$ "best guess" } & \multicolumn{2}{|c|}{$V^{*}[t]^{-1}:$ "precision" } \\
\hline & $\begin{array}{c}-8.088 \\
(-1.828)\end{array}$ & - & $\begin{array}{l}-0.2131 \\
(-1.48)\end{array}$ & - \\
\hline own-term & $\begin{array}{c}1.126 \\
(16.69)^{* *}\end{array}$ & $\begin{array}{c}1.002 \\
(320.6)^{* *}\end{array}$ & $\begin{array}{c}0.6696 \\
(32.11)^{* *}\end{array}$ & $\begin{array}{c}0.6632 \\
(32.47)^{* *}\end{array}$ \\
\hline gov-term & $\begin{array}{c}1.142 \\
(17.07)^{* *}\end{array}$ & $\begin{array}{c}1.020 \\
(442.5)^{* *}\end{array}$ & $\begin{array}{l}0.09493 \\
(1.85)^{*}\end{array}$ & $\begin{array}{l}0.04400 \\
(1.15)\end{array}$ \\
\hline env-term & $\begin{array}{c}1.110 \\
(16.79)^{* *}\end{array}$ & $\begin{array}{r}0.9894 \\
(228.8)^{* *}\end{array}$ & $\begin{array}{l}0.1668 \\
(1.54)\end{array}$ & $\begin{array}{l}0.07951 \\
(0.87)\end{array}$ \\
\hline $\begin{array}{l}\mathrm{H}_{0} \text { : Bayesian? } \\
\text { (F-test P-value) }\end{array}$ & 0.00000 & 0.00000 & 0.00000 & 0.00000 \\
\hline
\end{tabular}

a Let $D=\left(V[t]_{o}\right)^{-1}+\left(V[t]_{g}\right)^{-1}+\left(V[t]_{e}\right)^{-1}$. Then the "own-term," "gov-term," and "env-term" variables correspond to the first, second, and third constructed variables in each of the two equations:

$$
\begin{aligned}
& E *[t]=\alpha_{0}+\alpha_{1} \frac{\left(V[t]_{o}\right)^{-1} E[t]_{o}}{D}+\alpha_{2} \frac{\left(V[t]_{g}\right)^{-1} E[t]_{g}}{D}+\alpha_{3} \frac{\left(V[t]_{e}\right)^{-1} E[t]_{e}}{D}+\varepsilon_{E} \\
& V^{*}[t]^{-1}=\beta_{0}+\beta_{1}\left(V[t]_{o}\right)^{-1}+\beta_{2}\left(V[t]_{g}\right)^{-1}+\beta_{3}\left(V[t]_{e}\right)^{-1}+\varepsilon_{P}
\end{aligned}
$$


Table 3: Simple Linear Models for the Formulation of Posterior Subjective Distribution of Future Annual Average Temperatures $(n=608)$

\begin{tabular}{|c|c|c|c|c|}
\hline \multirow{2}{*}{$\begin{array}{l}\text { VARIABLE }^{\mathrm{a}} \\
\text { constant }\end{array}$} & \multicolumn{2}{|c|}{$E^{*}[t]:$ "best guess" } & \multicolumn{2}{|c|}{$V^{*}[t]:$ "variance" } \\
\hline & $\begin{array}{l}-20.58 \\
(-2.67)^{* *}\end{array}$ & - & $\begin{array}{l}0.08122 \\
(0.54)\end{array}$ & - \\
\hline own-term & $\begin{array}{c}0.4357 \\
(22.63) * *\end{array}$ & $\begin{array}{c}0.4258 \\
(22.43)^{* *}\end{array}$ & $\begin{array}{c}0.2986 \\
(15.76) * *\end{array}$ & $\begin{array}{c}0.3021 \\
(17.06)^{* *}\end{array}$ \\
\hline gov-term & $\begin{array}{c}0.5570 \\
(5.15) * *\end{array}$ & $\begin{array}{c}0.2877 \\
(7.33)^{* *}\end{array}$ & $\begin{array}{c}0.2593 \\
(2.80)^{* *}\end{array}$ & $\begin{array}{c}0.2899 \\
(3.99)^{* *}\end{array}$ \\
\hline env-term & $\begin{array}{c}0.3168 \\
(8.80)^{* *}\end{array}$ & $\begin{array}{c}0.2854 \\
(8.34)^{* *}\end{array}$ & $\begin{array}{c}0.2987 \\
(13.73)^{* *}\end{array}$ & $\begin{array}{c}0.3049 \\
(16.62)^{* *}\end{array}$ \\
\hline Test: & $\begin{array}{c}\alpha_{0}^{\prime}=0 \& \\
\alpha_{1}^{\prime}+\alpha_{2}^{\prime}+\alpha_{3}^{\prime}=1\end{array}$ & $\alpha_{1}^{\prime}+\alpha_{2}^{\prime}+\alpha_{3}^{\prime}=1$ & $\begin{array}{c}\beta_{0}^{\prime}=0 \& \\
\beta_{1}^{\prime}+\beta_{2}^{\prime}+\beta_{3}^{\prime}=1\end{array}$ & $\beta_{1}^{\prime}+\beta_{2}^{\prime}+\beta_{3}^{\prime}=1$ \\
\hline $\mathrm{H}_{0}$ : P-values & 0.0196 & 0.377 & 0.208 & 0.090 \\
\hline
\end{tabular}

a The "own-term," "gov-term," and "env-term" variables correspond to the first, second, and third variables in the following specifications:

$$
\begin{gathered}
E^{*}[t]=\alpha_{0}^{\prime}+\alpha_{1}^{\prime} E[t]_{o}+\alpha_{2}^{\prime} E[t]_{g}+\alpha_{3}^{\prime} E[t]_{e}+\varepsilon_{E}^{\prime} \\
V^{*}[t]=\beta_{0}^{\prime}+\beta_{1}^{\prime} V[t]_{o}+\beta_{2}^{\prime} V[t]_{g}+\beta_{3}^{\prime} V[t]_{e}+\varepsilon_{V}^{\prime}
\end{gathered}
$$


Table 4

Two-Equation Simultaneous Nonlinear Least Squares Model with Homogeneous Ambiguity Effects. $E^{*}[t]$ and $V^{*}[t]$ each a Weighted Average of Own, Government, and Environmental Group Analogs $(n=608$; Maximized Log $L=-2096.567)$

\begin{tabular}{|c|c|c|c|c|c|c|}
\hline \multirow[t]{2}{*}{ VARIABLE } & \multicolumn{3}{|c|}{$E^{*}[t]:$ "best guess" a } & \multicolumn{3}{|c|}{$V^{*}[t]:$ "variance" a } \\
\hline & $\kappa_{E}$ & $\gamma_{g}$ & $\gamma_{e}$ & $\kappa_{V}$ & $\delta_{g}$ & $\delta_{e}$ \\
\hline constant & $\begin{array}{c}0.2667 \\
(2.75)^{* *}\end{array}$ & $\begin{array}{c}1.708 \\
(2.15)^{* *}\end{array}$ & $\begin{array}{c}3.682 \\
(3.34)^{* *}\end{array}$ & $\begin{array}{c}0.9718 \\
(3.10)^{* *}\end{array}$ & $\begin{array}{c}8.537 \\
(3.66)^{* *}\end{array}$ & $\begin{array}{c}0.7414 \\
(2.95)^{* *}\end{array}$ \\
\hline AGE & - & - & $\begin{array}{c}-0.1975 \\
(-3.52) * *\end{array}$ & - & $\begin{array}{c}-0.4053 \\
(-3.78)^{* *}\end{array}$ & - \\
\hline FEMALE & - & $\begin{array}{c}0.6327 \\
(2.54)^{* *}\end{array}$ & $\begin{array}{c}0.7812 \\
(4.14)^{* *}\end{array}$ & - & $\begin{array}{c}1.678 \\
(5.26)^{* *}\end{array}$ & $\begin{array}{c}0.8221 \\
(5.25)^{* *}\end{array}$ \\
\hline INFORM & - & $\begin{array}{c}-0.4007 \\
(-3.16)^{* *}\end{array}$ & - & - & - & - \\
\hline CONSRV & - & $\begin{array}{c}0.2035 \\
(1.83)^{*}\end{array}$ & - & - & $\begin{array}{c}-0.1976 \\
(-1.74)^{*}\end{array}$ & - \\
\hline IMMOBILE & - & $\begin{array}{c}0.1930 \\
(2.51)^{* *}\end{array}$ & - & - & - & - \\
\hline RES.BIAS & - & $\begin{array}{c}-0.3394 \\
(-3.25)^{* *}\end{array}$ & - & - & - & - \\
\hline SMPL2 & - & - & $\begin{array}{c}-0.6324 \\
(-2.81)^{* *}\end{array}$ & - & - & - \\
\hline SMPL3 & - & $\begin{array}{l}-1.055 \\
(-3.52)^{* *}\end{array}$ & - & - & - & - \\
\hline
\end{tabular}

${ }^{a}$ These estimates correspond to the specification detailed in equations (6) and (7). In these specifications, the ambiguity effects parameters, $\kappa_{E}$ and $\kappa_{V}$ are simple scalars, but each of the four weight-related parameters, $\gamma_{g}, \gamma_{e}, \delta_{g}$ and $\delta_{e}$ is systematically varying. 


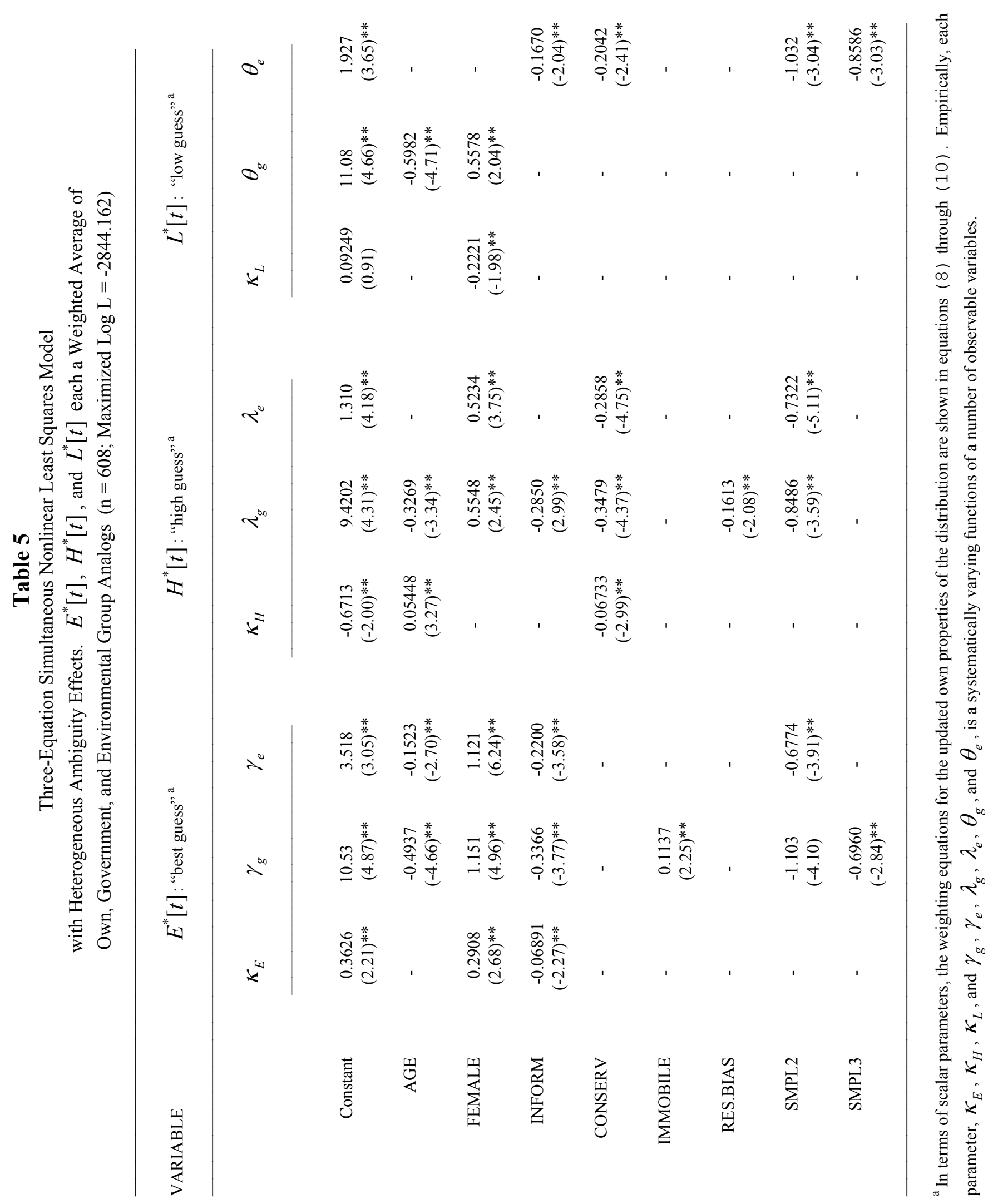




\section{Appendix}

\section{Randomized Design Characteristics of the Outside Information Sets Presented to Respondents}

Note that the historical annual average local temperature $=63.5$ degrees Fahrenheit.

A. Most likely future annual average temperature locally (Fahrenheit degrees)

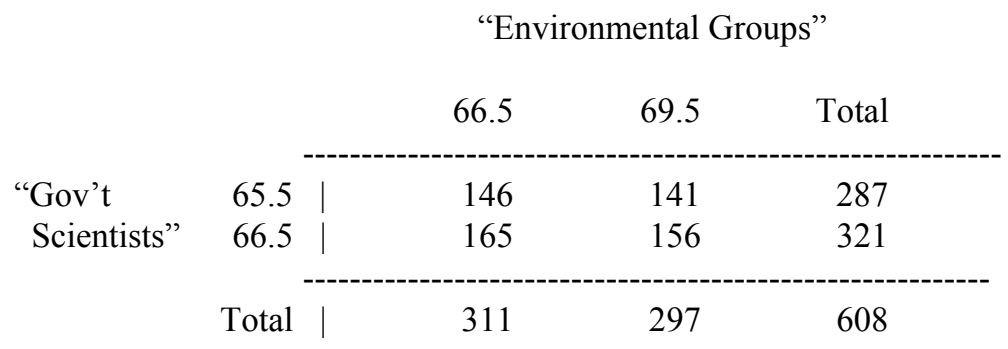

B. Lower bound on likely range of future annual average temperature locally (Fahrenheit degrees)

\begin{tabular}{|c|c|c|c|c|c|}
\hline & \multicolumn{5}{|c|}{ "Environmental Groups" } \\
\hline & & 63.5 & 65 & 66.5 & Total \\
\hline & 63.5 & 146 & 76 & 68 & 290 \\
\hline “Gov’t & 64.5 & 85 & 35 & 34 & 154 \\
\hline Scientists" & 65 & 86 & 42 & 36 & 164 \\
\hline & Total & 317 & 153 & 138 & 608 \\
\hline
\end{tabular}

C. Upper bound on likely range of future annual average temperature locally (Fahrenheit degrees)

\begin{tabular}{|c|c|c|c|c|c|c|}
\hline & & & "Envi & atal Gr & & \\
\hline & & 68 & 69.5 & 72.5 & 75.5 & Total \\
\hline & 66.5 & 35 & 43 & 34 & 42 & 154 \\
\hline "Gov’t & 67.5 & 35 & 33 & 28 & 37 & 133 \\
\hline Scientists" & 68 & 42 & 46 & 36 & 40 & 164 \\
\hline & 69.5 & 41 & 36 & 40 & 40 & 157 \\
\hline & Total | & 153 & 158 & 138 & 159 & 608 \\
\hline
\end{tabular}

Remark: After the survey, each participant group received an extensive lecture-long classroom debriefing about the purpose of the survey, its experimental design, and plans for the analysis of the survey data. Drawn specifically to their attention was the fact that these "outside information" sources were varied across respondents, and were (in some cases) somewhat extreme representations of the opinions of these unspecified authorities. 


\section{NOTES}

* As this project has evolved, it has benefited from comments received at presentations at the CERE/NOAA Conference on Research Transformations in Environmental Economics: Policy Design in Response to Global Change at Duke University (May 5-6, 1997) and at the EML/NSF Symposium on Preference Elicitation at UC Berkeley (July, 1997), the World Congress of Environmental and Resource Economists, Venice, Italy (June, 1998), and the Canadian Resource and Environmental Economics meeting, Guelph, Ontario, Canada (October, 2000). Data collection was facilitated by Kathleen McGarry, Fred Luk, Michael Sproul, Ed McDevitt, and Pari Kasliwal. Thanks are due to Vic Adamowicz, Anna Alberini, Ryan Bosworth, Graham Crawford, JR DeShazo, Barri Grossman, Jack Hirshleifer, Kathleen McGarry, and V. Kerry Smith for helpful comments, to Sara Wong Chang for careful data entry, and to Casey Williams for assistance in assembling the survey instruments. Any remaining errors are my own.

1 For example, cigarette packages display a rotating set of warnings from the Surgeon General. Megan's Law provides information on the whereabouts of registered sex offenders. California's Proposition 65 mandates that businesses disclose hazardous substances used on their premises, and the EPA's Toxic Release Inventory makes the presence of pollution and its sources know to the community. Adler and Pittle (1984) address the issue of information provision as a substitute for regulation.

2 Tobacco companies sponsor research about the risks of tobacco use and publicize the results. Civic leaders in communities with high rates of pollution or crime launch appealing public relations campaigns to recruit or retain businesses.

${ }^{3}$ In economics, the topics of (i.) individuals' risk perceptions, (ii.) how these risk perceptions 
respond to information, and (iii.) the value of risk changes, have been fertile areas for research. Some representative studies include Smith and Desvousges (1987, 1988), Smith and Johnson (1988), Viscusi (1985a, 1985b), Viscusi and Magat (1987, 1992), Viscusi et al. (1986), and Viscusi and O'Connor (1984). . In the context of health risk assessment, Johnson and Slovic (1995) look at the problem of conveying uncertainties and the consequences for risk perception and trust. Sjoberg (2001) reports that trust and risk perception bear only a weak relationship because people believe that there are limits to what even experts can know. In almost all these cases, the risks under consideration are physical health or workplace risks. But "risk" can of course be defined more broadly to include preferences over uncertain outcomes more generally.

${ }^{4}$ The question of climate change mitigation falls into the class of policy problems involving the regulation of risk. The work of Slovic, Fischhoff, and Lichtenstein (1985) is relevant.

${ }^{5}$ For more general and comprehensive treatments of the topic of risk perception, see Sjoberg, (2000), or Slovic (2000).

${ }^{6}$ For example, the issue of long-term environmental risks is addressed in Fischhoff (1990).

${ }^{7}$ Benson, Curley and Smith (1995) address the role of belief assessment in the process of eliciting probabilities. Williams and Hammitt (2001) elicit subjective risk perceptions for conventional and organic produce.

${ }^{8}$ Research currently in progress, employing an online survey, allows respondents to choose whether to view information from each source, on each of a variety of climate change topics, and to choose how much time to spend studying this information. In that case, exposure to 
information is endogenous, so the models for updating subjective probabilities concerning climate change will be much more complex.

${ }^{9}$ See also Heath and Tversky (1991).

${ }^{10}$ Viscusi and Magat's (1992) scenario is different in that they do not observe the individual's prior probability. They append a quadratic term in $R$ to their empirical model for a discrete prior probability, and this term can pick up any systematic shift in the implied prior probability due to the extent of the ambiguity in the external information.

${ }^{11}$ The larger project involves an online survey of students at many different institutions in conjunction with a general population mail survey, and is currently funded by the National Science Foundation (SBR 98-18875, 4/99-12/02).

${ }^{12}$ The first subsample (summer session 1997) employed no incentives and yielded a rather low response rate, as expected. The second subsample (Fall 1997) and third subsample (Fall 1998) were collected in the author's own Principles of Economics classes and Applied Regression Analysis classes. In these courses, the survey was integrated into the instruction in segments concerning the non-market valuation of public goods and the application of nonlinear simultaneous equations and probit models. Nominal extra credit was attached to submitting an anonymous survey and a detached "receipt" with the student's name and section number. This protocol was designed to preserve anonymity yet reward participation. The number of surveys collected was only slightly smaller than the number of receipts turned in, and only a handful of submitted survey instruments were turned in blank. Given the exploratory nature of the sample, we obviously did not (and could not) pursue non-response conversion. 
${ }^{13}$ The external information was protected during the elicitation of priors by sealing the centerfold of the survey instrument and announcing on the cover of the survey instrument "IMPORTANT: Do not break the seal on the center pages (or peek!) until you have finished Section B. Previewing this information may contaminate your answers to Section B." In the subsequent online version of the survey, this type of prohibition can be enforced. For the paperand-pencil version reported upon here, we must trust that most participants complied with this request.

${ }^{14}$ This information constitutes one component of the experimental design of the survey. All stylized forecasts fall within the range of assorted actual forecasts. Concerning survey research ethics, there is the delicate matter of not lying to respondents. Since we are purposefully vague about precisely which "government scientists" and "environmental groups" have made these forecasts, there may be some natural attenuation of the credibility assigned by respondents to these opinions. Respondents were debriefed thoroughly after the survey was collected.

${ }^{15}$ Research on the issue of whether individuals might be Bayesian decision-makers includes Viscusi (1985b), Viscusi and Magat (1992), Viscusi and O'Connor (1984), and Fischhoff and Beyth-Marom (1983).

${ }^{16}$ Quadratic terms in the disparity between the two outside information sources were not statistically significant in either equation in the systematically varying parameters model.

${ }^{17}$ Cvetkovich et al. (2002) focus on general trust in the nuclear power industry and the food supply industry. They find that individuals "low in general trust judged bad news as more 
informative than good news."

${ }^{18}$ This effect is observationally equivalent to the respondent's rejection of the survey's statement of environmental groups' opinions. Less attention may be paid to "outside opinions" if the survey's assertions are less credible to these respondents. 


\section{REFERENCES}

Adler, R.S. and Pittle, R.D. (1984). Cajolery or command: are education campaigns an adequate substitute for regulation? Yale Journal of Regulation 1 (2), 159-194.

Arrow, K.J. (1982). Risk perception in psychology and economics, Economic Inquiry 20, 1-9.

Arrow, K.J., Solow, R., Leamer, E.E., Portney, P., Radner, R., Schuman, H. (1993). Report of the NOAA panel on contingent valuation, Federal Register 58, 4601-4614.

Benson, P.G., Curley, S.P. and Smith, G.F. (1995). Belief assessment - an underdeveloped phase of probability elicitation. Management Science 41 (10), 1639-1653.

Bergstrom, J. and Stoll, J. (1990). An analysis of information overload with implications for survey design research. Leisure Science (12), 265-280.

Blomquist, G.C. and Whitehead, J.C. (1998). Resource quality information and validity of willingness to pay in contingent valuation. Resource and Energy Economics 20(2), 179-196.

Bohara, A. McKee, M. Berrens, R., Jenkins-Smith, H, Silva, C. and Brookshire, D. (1998). Effect of cost and group-size information on willingness to pay responses: open-ended vs. dichotomous choice. Journal of Environmental Economics and Management 35, 142-163.

Cameron, T.A. (2002). Individual Option Prices for Climate Change Mitigation. Manuscript, University of Oregon, Eugene, OR.

Cameron, T.A. and Englin, J.E. (1997). Respondent experience and contingent valuation of environmental goods. Journal of Environmental Economics and Management 33, 296-313.

Cvetkovich, G., Siegrist, M. Murray, R., and Tragesser, S. (2002). New information and social trust: Asymmetry and perseverance of attributions about hazard managers. Risk Analysis 22 (2), 359-367.

Davies, J.C., Covello, V.T. and Allen, F.W. (Eds) (1987). Risk Communication: Proceedings of the Conference on Risk Communication. Washington, D.C: The Conservation Foundation.

DeShazo, J.R., and Fermo, G. (2002). Designing choice sets for stated preference methods: the effects of complexity on choice consistency. Journal of Environmental Economics and Management. 44(1), 123-43.

Finucane, M.L.,Slovic P, Mertz CK, Flynn J, and Satterfield TA. (2000). Gender, race, and perceived risk: the 'white male' effect. Health Risk and Society. 2 (2), 159-172. 
Fischhoff, B. (1990). Understanding long-term environmental risks. Journal of Risk and Uncertainty 3, 315-30.

Fischoff, B. and Beyth-Marom, R. (1983). Hypothesis evaluation from a Bayesian perspective, Psychological Review 90, 239-60.

Flynn, J., Slovic P. and Mertz, C.K. (1994). Gender, race, and perception of environmentalhealth risks. Risk Analysis 14(6), 1101-1108.

Heath, C. and Tversky, A. (1991). Preference and belief: ambiguity and competence in choice under uncertainty. Journal of Risk and Uncertainty 4(1), 5-28.

Hoehn, J.P. and Randall, A. (2002). The effect of resource quality information on resource injury perceptions and contingent values. Resource and Energy Economics 24(1-2), 13-31.

Johnson, B.B. and Slovic, P. (1995). Presenting uncertainty in health risk assessment - initial studies of its effects on risk perception and trust. Risk Analysis 15 (4), 485-494.

Munro, A. and Hanley, N. (1999). Information, uncertainty, and contingent valuation, in Bateman, I. and Willis, K. (eds) Valuing Environmental Preferences: Theory and Practice of the Contingent Valuation Method in the US, EU and Developing Countries. Oxford: Oxford University Press.

Siegrist M., Cvetkovich, G.T., and Gutscher, H. (2001). Shared values, social trust, and the perception of geographic cancer clusters. Risk Analysis 21(6), 1047-1053.

Sjoberg, L. (2000). Factors in risk perception. Risk Analysis 20(1), 1-11.

Sjoberg, L. (2001). Limits of knowledge and the limited importance of trust. Risk Analysis 21(1), 189-198.

Slovic, P. (2000). The Perception of Risk, London: Earthscan.

Slovic, P., Fischhoff and B., Lichtenstein, S. (1985). Regulation of risk: a psychological perspective, in Noll, R. (Ed.) Regulatory Policy and the Social Sciences. University of California Press, Berkeley, CA.

Smith, V.K., Desvousges, W. (1987). An empirical analysis of the economic value of risk changes. Journal of Political Economy 95, 89-114.

Smith, V.K., and Desvousges, W. (1988). Risk perception, learning and individual behavior. American Journal of Agricultural Economics 70, 1113-17. 
Smith, V.K., and Johnson, F.R. (1988). How do risk perceptions respond to information? The case of radon. Review of Economics and Statistics 70 (1), 1-8.

Tversky, A. and Kahneman, D. (1974). Judgment under uncertainty: heuristics and biases, Science 185, 1124-31.

Viscusi, W.K. (1985a). A Bayesian perspective on biases in risk perception. Economics Letters $17,59-62$.

Viscusi, W.K. (1985b). Are individuals Bayesian decision makers? AEA Papers and Proceedings. 381-385.

Viscusi, W.K. and Magat, W.A. (1987). Learning about Risk: Consumer and Worker Responses to Hazard Information. Cambridge: Harvard University Press.

Viscusi, W.K. and Magat, W.A. (1992). Bayesian decisions with ambiguous belief aversion, Journal of Risk and Uncertainty 5, 371-387.

Viscusi, W.K., Magat, W.A., and Huber, J. (1986). Informational regulation of consumer health risks - An empirical evaluation of hazard warnings. Rand Journal of Economics, 351-365.

Viscusi, W.K., and O'Connor, C.J. (1984). Adaptive responses to chemical labeling: are workers Bayesian decision makers? American Economic Review, 942-956.

Williams, P.R.D., and Hammitt, J.K. (2001). Perceived risks of conventional and organic produce: Pesticides, pathogens, and natural toxins. Risk Analysis 21(2), 319-330. 
